\title{
Febuxostat: the evidence for its use in the treatment of hyperuricemia and gout
}

\author{
This article was published in the following Dove Press journal: \\ Core Evidence \\ 15 June 2009 \\ Number of times this article has been viewed
}

\author{
Angelo L Gaffo' \\ Kenneth G Saag ${ }^{2}$ \\ 'Birmingham VA Medical Center, \\ ${ }^{2}$ Division of Clinical Immunology \\ and Rheumatology, University \\ of Alabama at Birmingham
}

Introduction: Gout is a common and disabling cause of arthritis in middle-aged and elderly populations, with its main predisposing factor being hyperuricemia (serum urate $>6.8 \mathrm{mg} / \mathrm{dL}$ ). Options for treatment of chronic gout until 2008 were allopurinol, a xanthine oxidase inhibitor, and the group of drugs known as uricosurics that stimulate the renal excretion of uric acid. A proportion of patients, including some with chronic kidney disease and solid organ transplantations, could not be treated with the those therapies because of intolerance, drug interactions, or adverse events. Febuxostat is a nonpurine xanthine oxidase inhibitor, recently approved in Europe and the United States for the treatment of chronic gout.

Aim: To review the clinical evidence (phase II and III studies) of the effectiveness and safety of febuxostat for treatment of hyperuricemia and gout.

Evidence review: Febuxostat, at doses ranging from 40 to $240 \mathrm{mg} /$ day, is efficacious in reducing serum urate in patients with hyperuricemia and gout, comparing favorably with fixed doses of allopurinol in that respect. Early safety signals with respect to liver test abnormalities and cardiovascular outcomes have not been confirmed in recent large prospective trials but need to be further monitored.

Clinical potential: Given its low cost and extensive clinical experience, allopurinol will likely remain the first-line drug for management of hyperuricemia and gout. Febuxostat may provide an important option in patients unable to use allopurinol, those with very high serum urate levels, or in the presence of refractory tophi.

Keywords: febuxostat, gout, hyperuricemia, evidence

\section{Core evidence outcomes summary for febuxostat in hyperuricemia and gout}

\begin{tabular}{|c|c|c|}
\hline Outcome measure & Evidence & Implications \\
\hline \multicolumn{3}{|l|}{ Patient-oriented evidence } \\
\hline Reduction in gout flares & Moderate & $\begin{array}{l}\text { An expected increase in gout flares was shown with } \\
\text { initiation of treatment } \\
\text { A subsequent reduction with continuous therapy is } \\
\text { suggested by clinical trials but this has not been the } \\
\text { primary outcome in any study } \\
\text { No superiority compared to allopurinol has been } \\
\text { demonstrated }\end{array}$ \\
\hline $\begin{array}{l}\text { Reduction in tophi numbers } \\
\text { or volume }\end{array}$ & Moderate & $\begin{array}{l}\text { Studies and abstracts suggest a reduction in tophi size, } \\
\text { limited evidence of superiority against allopurinol in } \\
\text { this respect }\end{array}$ \\
\hline $\begin{array}{l}\text { Drug is free of serious } \\
\text { adverse effects }\end{array}$ & Moderate & $\begin{array}{l}\text { Important safety signals raised by clinical studies have } \\
\text { not been confirmed in recent large prospective trials } \\
\text { Postmarketing surveillance will be required }\end{array}$ \\
\hline
\end{tabular}

(Continued)
Correspondence: Kenneth G Saag

FOT 820,1530 3rd Ave

S Birmingham, AL 35294, USA

Email ksaag@uab.edu. submit your manuscript $\mid$ www.dovepress.com

Dovepress 


\begin{tabular}{|lll|}
\hline (Continued) & & \\
\hline Outcome measure & Evidence & Implications \\
\hline $\begin{array}{l}\text { Improvement in quality of life } \\
\text { Disease-oriented evidence } \\
\text { Effective control } \\
\text { of hyperuricemia }\end{array}$ & No evidence & \\
& Clear & $\begin{array}{l}\text { Febuxostat is effective in reducing serum urate levels } \\
\text { in patients with hyperuricemia and gout } \\
\text { It compares favorably at multiple dosages with } \\
\text { fixed-dose allopurinol (300 mg/day) }\end{array}$ \\
$\begin{array}{l}\text { Economic evidence } \\
\text { Cost effectiveness as } \\
\text { treatment of hyperuricemia } \\
\text { and gout }\end{array}$ & Limited & $\begin{array}{l}\text { Incremental cost-effectiveness ratio } £ 15 \text { 565-16 } 574 \\
\text { (\$US24 342-25 920) per quality-adjusted life-year, }\end{array}$ \\
& & $\begin{array}{l}\text { but acquisition costs likely to be much higher than } \\
\text { generic allopurinol }\end{array}$ \\
\hline
\end{tabular}

\section{Scope, aims, and objectives}

The aim of this article is to review the clinical evidence of the effectiveness of febuxostat (TEI-6720, or TMX-67) on outcomes and its potential for clinical management of hyperuricemia and gout.

\section{Methods}

This review analyzes phases II and III evidence of febuxostat in the management of gout. Literature searches were performed using PubMed (www.pubmed.gov 1950-November 2008) and the Cochrane database (www.cochrane.org) with the search terms “febuxostat" OR “TEI-6720” OR “TMX-67” (Table 1):

- 75 references were identified

- eight were clinical trials

- five phase I studies, one phase II trial, and two phase III trials.

Additionally, abstracts from the American College of Rheumatology meetings (available at www.rheumatology.org,

Table I Evidence base included in the review

\begin{tabular}{lll}
\hline Category & \multicolumn{2}{l}{ Number of records } \\
\cline { 2 - 3 } & Full papers & Abstracts \\
\hline Initial search & 67 & 23 \\
Records excluded & 65 & $1 \mathrm{I}$ \\
Records included & 2 & 12 \\
Additional studies identified & 0 & 0 \\
Level I clinical evidence & 0 & 0 \\
Level 2 clinical evidence & 2 & 3 \\
Level $\geq 3$ clinical evidence & 0 & 9 \\
Studies other than RCT & & 9 \\
Case reports & & 0 \\
Economic evidence & 0 & 0 \\
\hline
\end{tabular}

Notes: apen label or nonrandomized studies conducted with data collected from phase II or III clinical trials; published only in abstract form.

For definitions of levels of evidence see Core Evidence website (http://www.dovepress. com/core-evidence-journal).

Abbreviation: RCT, randomized controlled trial. years 2001-2008) and European League Against Rheumatism meetings (available at www.eular.org, years 2001-2008) were browsed using the terms "febuxostat" OR "TEI-6720" OR "TMX-67":

- 24 abstracts were identified

- seven were phase II or III clinical trials

- six abstracts were ancillary studies, performed with data previously collected on phase II or III trials

- three abstracts were extensions of phase II or III trials.

Ongoing or unfinished studies were searched from the federal database (www.clinicaltrials.gov). Only one study, recently completed and presented at the meeting of the American College of Rheumatology was identified. The economic evidence from the report of the National Institute of Clinical Excellence (NICE) from the UK (www.nice. org.uk) is also discussed.

\section{Disease overview}

Uric acid (or urate) is the end product of purine metabolism in humans and several other higher primates. Concentrations of urate beyond its limit of solubility in serum $[6.8 \mathrm{mg} / \mathrm{dL}$ at $\left.37^{\circ} \mathrm{C}(404 \mu \mathrm{mol} / \mathrm{L})\right]$ defines hyperuricemia, ${ }^{1}$ a necessary but not sufficient factor for the development of gout, the disease state characterized by tissue deposition of monosodium urate (MSU) crystals and its associated symptoms.

Gout is a common diagnosis in Western populations. According to the most recent estimate by the National Arthritis Data Workgroup using 1996 data from the National Health Interview Survey (NHIS) and National Health and Nutrition Examination Survey (NHANES) in the US, 3 million adults older than 18 years had gout in the past year and 6.1 million adults over 20 years old have been diagnosed with gout at some point in their lives. ${ }^{2}$ The incidence of gout has been increasing in the US ${ }^{3}$ and is currently considered the most common inflammatory arthritis in men over 40 years old, exceeding rheumatoid arthritis. ${ }^{2}$ Worldwide, gout frequencies 
and time trends are heterogeneous with high prevalence in specific populations like Malayo-Polynesians $(1.7 \%)^{4}$ and New Zealand Maoris (8.8\%). ${ }^{5}$

A definitive diagnosis of gout is confirmed by the observation of intracellular needle-shaped, negatively birefringent crystals on synovial fluid aspirated from an affected joint or the presence of tophi (tissue deposits of crystallized monosodium urate that are usually evident under the skin of patients with chronic hyperuricemia). The disease is characterized by recurrent attacks of pain and swelling in the affected joints. If the hyperuricemia underlying the disease is not treated, the disease can progress into a chronic stage leading to chronic pain, significant impairment in quality of life, and disability. ${ }^{6-8}$ Clinical and radiographic features can contribute to the diagnosis in cases with atypical presentations. The burden of disease is considerable in patients with solid organ transplants, mainly because of the hyperuricemic effect of cyclosporine. ${ }^{9}$ Affected patients frequently suffer from multiple comorbidities and polypharmacy, making management of acute flares and chronic hyperuricemia difficult because of intolerances to first-line agents and drug interactions.

\section{Current therapy options for chronic gout}

The management goals in gout differ depending on the setting and stage of the disease. In acute gout pharmacologic treatment is aimed at resolving the prominent pain and inflammation. In the intercritical periods (asymptomatic periods in between gout flares) the goal is to maintain serum urate at subsaturation levels preventing the development of tophi, and preventing the recurrence of new attacks through prophylactic management. A list of the agents currently available for management of gout is presented in Table 2.

\section{Pharmacologic options for chronic hyperuricemia and gout}

This section deliberately excludes a discussion on febuxostat, that will be discussed extensively in the following sections. The decision about initiating long term therapy for hyperuricemia and gout prevention should be individualized. Currently, there is no evidence that treating asymptomatic hyperuricemia alone is efficacious or cost effective. However, very high levels of serum urate place individuals at a very high risk for incident gout ${ }^{10}$ and, at a minimum, lifestyle changes including reduction in alcohol intake, dietary changes, and weight loss should be considered. Additionally, a reassessment of the need for other pharmacologic therapies that could be affecting serum urate levels like diuretics should be considered. ${ }^{11,12}$ After a single episode of acute gout, long-term urate-lowering therapy may not yet be indicated, as joint damage is unlikely to occur in patients who remain asymptomatic. However, there is reasonable consensus that long-term therapy is advocated in patients with two or more flares per year or with tophi. ${ }^{11-14}$

The goal of chronic urate-lowering therapy should be to reduce serum urate until the goal of a subsaturation concentration of uric acid at or about $6.8 \mathrm{mg} / \mathrm{dL}$ or less is reached (a more ambitious goal of $6.0 \mathrm{mg} / \mathrm{dL}$ or less is recommended according to recent guidelines). ${ }^{11}$ Hyperuricemia develops secondary to either overproduction (excretion of more than $600 \mathrm{mg} /$ day in the urine; accounting for $10 \%-15 \%$ of hyperuricemia cases) or underexcretion (excretion of less than $330 \mathrm{mg}$ /day; approximately $85 \%-90 \%$ of hyperuricemia cases). ${ }^{1}$ Both overproduction and underexcretion could be primary, such as in the inherited enzymatic disorders of urate production (hypoxanthine-guanine phosphoribosil transferase defect or phosphoribosilpyrophosphatase synthetase [PRPP] overactivity) or the condition known as familial juvenile hyperuricemic nephropathy. Common secondary or acquired causes of overproduction and underexcretion

Table 2 Therapeutic agents for management of gout

\begin{tabular}{ll}
\hline Acute gout flares & - Nonpharmacologic: icepacks \\
& - Nonsteroidal anti-inflammatory drugs \\
& - Colchicine \\
& - Glucocorticoids \\
Urate-lowering agents for chronic gout & - Cosyntropin (ACTH derivative) \\
& - Xanthine-oxidase inhibitor: allopurinol, febuxostat \\
& - Uricosuric agents: probenecid, sulfinpyrazone, benzbromarone \\
& - Other agents with urate-lowering properties: fenofibrate, losartan \\
\hline
\end{tabular}

Abbreviation: $\mathrm{ACTH}$, adrenocorticotropic hormone. 
are due to excessive purine turnover (diet, malignancies), medications, and toxins. Distinguishing between serum urate overproducing and underexcreting gout patients may have therapeutic significance but does not need to be routinely performed. ${ }^{15}$ Concomitant use of colchicine or NSAIDs during the initiation of urate-lowering may be needed to prevent rebound flares. ${ }^{11,16}$

\section{Xanthine oxidase inhibitors}

Allopurinol is the preferred urate-lowering agent in view of its efficacy in overproducers and underexcretors of serum urate, relatively easy dosing regimen, low cost, and generally acceptable safety profile. Allopurinol and its metabolite oxypurinol are both substrates and inhibitors of xanthine oxidase, blocking the conversion of hypoxanthine to xanthine to uric acid. ${ }^{17}$ Both lower serum and urinary urate levels. The recommended starting dose of allopurinol is $100 \mathrm{mg} /$ day with progressive increases in the dosage every 2-4 weeks until reaching a target of $300 \mathrm{mg} /$ day. ${ }^{11}$ However, it is common practice to start treatment fixed at dosages ranging from 50-300 mg/day depending on factors such as renal function, age, and comorbidities. Lower initial doses are preferred because an expected lower frequency of acute gout flares and hypersensitivity reactions after initiation of therapy. It is important to monitor serum urate levels regularly for dosage adjustments until the target concentration is reached. There is no evidence about how often this monitoring should be done, but it makes sense to know the serum urate concentration before each dose increase during the titration phase to make sure the patient is not already at goal; a goal that some patients may reach with doses as low as 100-200 mg/day. ${ }^{18}$ Allopurinol should be started at a lower dose in elderly patients and those with impaired kidney function. However, renal adjusted doses usually fail to achieve target serum urate levels and continuous titration of the drug to the goal should be carefully attempted. ${ }^{19}$ The most commonly used allopurinol dose of $300 \mathrm{mg} /$ day achieves target serum urate concentrations in only $53 \%$ of patients ${ }^{20}$ and dosages as high as $800 \mathrm{mg} /$ day (the highest dose recommendation by the FDA) or even up to $1200 \mathrm{mg} /$ day are occasionally necessary. However, before escalating the dose to very high levels adherence should be assessed because as many of $50 \%$ of patients are noncompliant with the medication, ${ }^{21}$ especially if they are having recurrent gout flares. The major barrier to achieve optimal disease control with allopurinol is under dosing. This is possibly due to a lack of awareness of target serum urate and concerns about the safety of the drug in the primary care setting, where most of the gout care is usually provided. ${ }^{22,23}$

Adverse reactions from allopurinol are generally uncommon and most are mild. The most frequent toxicities are rash, gastrointestinal intolerance such as diarrhea, headache, and leukopenia. Rashes can be recurrent on reexposure to the drug and an important cause of intolerance. Although in general very rare, allopurinol exposure is one of the most common causes of toxic epidermal necrolysis and Stevens-Johnson syndrome. ${ }^{24}$ Given the scarcity of treatment alternatives for certain patients, allopurinol desensitization protocols have been developed. ${ }^{25}$ The allopurinol hypersensitivity syndrome is an uncommon immune-mediated severe reaction with mortality of up to $20 \%$. It is characterized by fever, rash, acute renal insufficiency, eosinophilia, hepatic injury, and vasculitis. Renal impairment, diuretic use, and recent initiation of allopurinol have been implicated in the development of allopurinol hypersensitivity syndrome. ${ }^{26-29}$ In a case series of 78 patients with a creatinine clearance of less than $10 \mathrm{~mL} / \mathrm{min}$ there was virtually no renal excretion of the main allopurinol metabolite, oxypurinol. ${ }^{28}$ Along the same line, cases of allopurinol hypersensitivity have been linked to $\mathrm{T}$ cell mediated immune reactions to oxypurinol, ${ }^{30}$ possibly induced by certain human leukocyte antigen (HLA) haplotypes like the strong association described with HLAB5801 in Han Chinese population. ${ }^{31}$

Multiple drug interactions may be an additional factor limiting the use of allopurinol; notably allopurinol can increase drug levels of theophylline, warfarin, and azathioprine, the latter regarded, except in rare instances, a contraindication. ${ }^{32}$ Thiazide diuretics can inhibit the excretion of allopurinol and potentiate allopurinol toxicity. ${ }^{13}$ Finally, a high incidence of skin rashes has been described with the combination of ampicillin or amoxicillin with allopurinol. ${ }^{33}$

\section{Uricosuric agents}

Uricosuric drugs reverse the most common physiologic abnormality in gout, namely, underexcretion of uric acid (less than $330 \mathrm{mg}$ of uric acid/24 hours on a regular diet). Probenecid and sulfinpyrazone are used internationally, with benzbromarone (never available in the US) being recently withdrawn from the market in the countries where it was available by its principal manufacturer, because of hepatic toxicity. ${ }^{34}$ Other drugs with mild uricosuric effects include losartan and fenofibrate. Uricosuric agents probably act at the level of the URAT-1 transporter in the proximal tubule. ${ }^{35}$ When 
used on ideal patient candidates, probenecid, sulfinpyrazone, and benzbromarone can be successful $70 \%-80 \%$ of the time in achieving optimal serum urate levels. ${ }^{36}$ However, several limitations are encountered when trying to use uricosurics in practice. First, they rapidly lose effectiveness as the glomerular filtration rate drops below $50 \mathrm{~mL} / \mathrm{min}$. A potential exception to this rule is benzbromarone, which can remain effective in mild to moderate renal impairment at the cost of an increased incidence of hepatic toxicity. ${ }^{34}$ Second, their use is strongly discouraged in patients with history of renal calculi as the uricosuric agents may further promote nephrolithiasis. Lastly, their use is not recommended in elderly patients, those on multiple medications (because of multiple drug interactions), or in patients who have trouble complying with multiple daily doses. For example, probenecid has known interactions with azathioprine, rifampin, salicylates, penicillins, indomethacin, and heparin. ${ }^{36}$ Probenecid is the most widely used uricosuric; usually initiated at a dose of $500 \mathrm{mg}$ orally twice a day, the dosage can be slowly increased up to $3 \mathrm{~g} /$ day. Adverse effects include gastrointestinal intolerance, rash, hepatotoxicity, acute gout attacks, nephrolithiasis, and nephrotic syndrome. ${ }^{37,38}$

\section{Unmet needs with older therapies}

Few drugs are available for long-term urate-lowering therapy. Uricosurics are of limited application because of the high frequency of comorbidities (particularly renal, which render their use ineffective) and drug interactions, significantly limiting their use in real clinical practice. Only one approved drug, allopurinol, targets the overproduction mechanism of hyperuricemia and its use is limited in some cases by drug interactions, intolerance, and adverse reactions, one of which, the allopurinol hypersensitivity syndrome, is very serious.

There are a number of patients with gout in whom no approved alternative for management of their chronic hyperuricemia is available because of allopurinol or uricosuric intolerances, allergies, or ineffectiveness. A significant proportion of these patients have kidney dysfunction or solid organ transplantations, with the management of the disease being limited only to relief of persistent attacks. Some of these patients have chronic tophaceous gout that requires long-term glucocorticoids, with its known metabolic and bone health consequences, besides the negative impact on quality of life.

There has been a clear need for more alternatives in patients with gout in whom allopurinol is contraindicated or not tolerated. Until February 2009, no new drugs had been approved for gout in the US since allopurinol was introduced in 1964.

\section{Clinical evidence of the efficacy of febuxostat in the treatment of gout}

Febuxostat is an orally administerd, nonpurine selective inhibitor of xanthine oxidase. The drug acts by binding into a channel in the molybdenum center of the enzyme, leading to a very stable and long-lived enzyme-inhibitor interaction with both the oxidized and reduced forms of the enzyme and, as a consequence, a strong inhibition of substrate binding. ${ }^{39}$

\section{Phase II data}

Only one phase II study has been published investigating febuxostat. ${ }^{40}$ Three ancillary studies and two reports of openlabel extensions of this trial are available in abstract form only. One other phase II study from Japan is available also only in abstract form.

Becker et $\mathrm{l}^{40}$ conducted a 28 -day, multicenter, doubleblind, placebo-controlled, dose response clinical trial to determine the safety and efficacy of once daily febuxostat (at doses of 40, 80, and $120 \mathrm{mg}$ ) in reducing serum uric acid (SUA) in patients with American College of Rheumatology criteria-defined gout aged 23-80 years old. Several exclusion criteria, including absence of patients with kidney dysfunction or taking drugs known to affect serum urate (such as aspirin or diuretics), made this a selected population with limited generalizability. A total of 153 patients were finally enrolled and randomly assigned to placebo or one of the three study drug arms.

A greater proportion of patients in the three febuxostat arms achieved target SUA levels of $6.0 \mathrm{mg} / \mathrm{dL}$ or less. In most cases this reduction was seen as early as 7 days after starting the drug and maintained throughout the study period. There was a dose-dependent effect of febuxostat, with more patients assigned to the higher doses of the drug achieving SUA of $5.0 \mathrm{mg} / \mathrm{dL}$ and $4.0 \mathrm{mg} / \mathrm{dL}$. As expected and possibly as a consequence of sudden removal and mobilization of uric acid crystals from the tissues, the incidence of gout flares, despite pretreatment with colchicine, was higher in the febuxostat $80 \mathrm{mg} /$ day and $120 \mathrm{mg} /$ day arms than with placebo. Diarrhea and abdominal pain were side effects reported in patients taking febuxostat, but the frequency was not different from that with placebo. Abnormal liver function test results were reported in five (14\%), three ( $8 \%$ ), and three (8\%) of the patients taking febuxostat 40,80 , and $120 \mathrm{mg} /$ day, respectively. The authors concluded that there was a prompt and persistent efficacy of febuxostat in lowering SUA concentrations in patients with hyperuricemia and gout. 
The three ancillary studies based on this study and published only in abstract form described reductions in tophi volume (measured by magnetic resonance imaging) in nine patients with decreased SUA levels while on febuxostat, ${ }^{41}$ good tolerance of febuxostat in allopurinol-intolerant patients, ${ }^{42}$ and need for at least 3 months colchicine prophylaxis in patients starting febuxostat. ${ }^{43}$ Two abstract reports of openlabel extensions up to 48 months describe continuous efficacy of febuxostat in achieving and maintaining an SUA of less than $6.0 \mathrm{mg} / \mathrm{dL}$, along with a reduction in gout flares and tophi. Adverse reactions mentioned include diarrhea, gastrointestinal motility disorders, headache, abnormal liver function tests, and hyperlipidemia. ${ }^{44,45}$ No descriptions of the frequencies of these reactions were provided.

A phase II abstract from Japan reported a trial in which 128 patients with gout and/or hyperuricemia were randomized to placebo or one of three febuxostat dosages (10, 20, or $40 \mathrm{mg} /$ day). ${ }^{46}$ All dosages of febuxostat reduced SUA in patients with gout and/or hyperuricemia, regardless of whether they were underexcretors or overproducers of serum urate. Febuxostat was reported to be safe and well tolerated, with main adverse effects being abnormal liver function tests and gout flares.

\section{Phase III data}

Two phase III trials have been published in peer-reviewed journals and one has recently become available in abstract form only. Three ancillary studies and an open-label extension of these trials are available in abstract form only. Two phase III studies from Japan are also available only in abstract form.

The Febuxostat versus Allopurinol Controlled Trial $(\text { FACT })^{47}$ was a randomized, double-blind, 52-week, multicenter trial aimed at evaluating the efficacy and safety of febuxostat 80 and $120 \mathrm{mg}$ once daily compared with allopurinol at a fixed dose of $300 \mathrm{mg}$ /day in adult patients with American College of Rheumatology-defined gout and a SUA concentration of at least $8.0 \mathrm{mg} / \mathrm{dL}(480 \mu \mathrm{mol} / \mathrm{L})$. Main exclusion criteria included kidney dysfunction, concomitant drugs known to affect serum urate (eg, urate-lowering agents, aspirin, or diuretics), very high body mass index $(>50)$, active liver disease, pregnancy, use of prednisone at more than $10 \mathrm{mg} /$ day, or alcohol abuse. The primary endpoint was the achievement of an SUA concentration of $6.0 \mathrm{mg} / \mathrm{dL}$ or less at each of the last three monthly evaluations. Secondary endpoints included achievement of an SUA of $6.0 \mathrm{mg} / \mathrm{dL}$ or less at each visit and the percentage reduction in SUA concentration at each visit. Clinical endpoints included reduction in tophus area, change in number of tophi, and proportion of patients requiring treatment for acute gout flares from weeks 9 to 52 .

A total of 1283 patients were screened and 762 were randomized to allopurinol or one of the two febuxostat arms. Prophylaxis of gout flares with colchicine or naproxen was administered to all patients during a 2 -week washout period and the first 8 weeks of the double-blind treatment period. There were no significant differences between groups after randomization, with a strong predominance of middle-aged (mean age 51-52 years old) white patients (75\%-79\% of patients in all groups). Mean SUA ranged from 9.80 to $9.90 \mathrm{mg} / \mathrm{dL}$ and years since gout diagnosis from 11.5 to 12.6 years. A larger proportion of patients in the febuxostat arms than in the allopurinol arm discontinued the interventions before study completion: 88 (34\%) with febuxostat $80 \mathrm{mg} /$ day, 98 (39\%) with febuxostat $120 \mathrm{mg} /$ day, and $66(26 \%)$ with allopurinol. Most of these discontinuations were due to losses to follow-up, but higher proportions of adverse events and gout flares leading to discontinuation were seen with febuxostat compared with allopurinol.

The primary endpoint of achievement of a SUA of $6.0 \mathrm{mg} / \mathrm{dL}$ or less in the last three visits was significantly more frequent in the two febuxostat arms $(53 \%$ of patients in the $80 \mathrm{mg} /$ day arm and $62 \%$ of patients in the $120 \mathrm{mg} /$ day arm) compared with allopurinol (21\%). The secondary endpoints were also significantly more frequently achieved in the febuxostat arms. There were significantly larger proportions of patients suffering from at least one gout flare from the beginning of therapy through week 8 in the febuxostat $120 \mathrm{mg}$ /day group (36\%) compared with allopurinol (21\%), but these proportions became similar in the analysis from week 9 through week 52 (range 64\%-70\%). The incidence of flares reduced gradually; by week 49-52, the final visit interval, the incidence was $8 \%$ with febuxostat $80 \mathrm{mg}, 6 \%$ with febuxostat $120 \mathrm{mg}$ and $11 \%$ with allopurinol. There was no significant difference in the reduction in tophi areas between groups.

The rates of total adverse events and serious adverse events were reported as similar between the groups. Most frequently reported adverse events were liver function test abnormalities (range 4\%-5\%), diarrhea (3\% in all three groups), and headaches (range from $1 \%-3 \%$ ). It is very important to note that two patients in the febuxostat $80 \mathrm{mg} /$ day arm and two in the febuxostat $120 \mathrm{mg} /$ day arm died during the study period (two from cardiovascular events) versus none in the allopurinol arm. These events were considered unrelated to the administration of the study 
medication by the investigators. The authors' conclusion was that all urate-lowering endpoints requiring an SUA concentration of $6.0 \mathrm{mg} / \mathrm{dL}$ or less were achieved by more febuxostat than allopurinol recipients, but the clinical outcomes (reduction in gout flares and tophi area) were not different.

A second phase III trial of febuxostat has been recently published and expanded upon the results of the FACT study. Named Allopurinol and Placebo-Controlled, Efficacy Study of Febuxostat (APEX), it was a randomized, double-blind, allopurinol and placebo-controlled trial that followed 1072 subjects for 28 weeks. ${ }^{48}$ The objective of the study was to compare the safety and efficacy of febuxostat at dosages of 80,120 , and $240 \mathrm{mg} /$ day with allopurinol or placebo. Additionally, an assessment of the effects of treatment with febuxostat in patients with impaired renal function was sought, and those with mild to moderate renal dysfunction (serum creatinine up to $2.0 \mathrm{mg} / \mathrm{dL}$ ) were allowed to participate in the study. The fixed doses in the allopurinolcontrolled arm were divided according to the renal function as follows: $300 \mathrm{mg} /$ day was administered to patients with serum creatinine up to $1.5 \mathrm{mg} / \mathrm{dL}$, and $100 \mathrm{mg} /$ day to patients with serum creatinine between $1.5-2 \mathrm{mg} / \mathrm{dL}$. To be eligible for the study, patients needed to be between 18 and 85 years of age, have American College of Rheumatology-defined gout, hyperuricemia with an SUA of $\geq 8.0 \mathrm{mg} / \mathrm{dL}$, and a creatinine level up to $2.0 \mathrm{mg} / \mathrm{dL}$ as described above. Exclusion criteria included intolerances to allopurinol, colchicine, or naproxen; history of renal calculi, heavy alcohol intake, baseline hepatic transaminases $\geq 1.5$ the upper limit of normal; and any other significant medical conditions as defined by the investigators. The study endpoints were similar to the ones described above for the FACT study.

Of the 1641 patients screened 1072 were finally randomized to the five arms of the study and the same number analyzed under an intention-to-treat protocol. A statistically larger number of premature withdrawals were found in the febuxostat $80 \mathrm{mg} /$ day and febuxostat $240 \mathrm{mg} /$ day arms, with the latter group having a larger number of withdrawals because of gout flares. The baseline characteristics of the patients showed a large majority of middle-aged white males (mean ages 51-54 years across groups). Important proportions of patients were obese (62\%) and hypertensive (33\%). The mean duration of gout was 10.9 years, and $20 \%$ of patients had tophi across the different randomized groups. The mean baseline serum urate was $9.85 \pm 1.26 \mathrm{mg} / \mathrm{dL}$. Only $4 \%$ of subjects had an impaired renal function (serum creatinine of $1.5-2 \mathrm{mg} / \mathrm{dL})$. Significantly greater proportions of patients in all the febuxostat arms achieved the primary study endpoint of having the last three SUA levels measured at $\leq 6.0 \mathrm{mg} / \mathrm{dL}$ when compared with either allopurinol or placebo. It was revealing that a small proportion of patients in the allopurinol arm achieved the primary goal $(\sim 20 \%$ of the overall intention-to-treat population), emphasizing the concept that a fixed dose of allopurinol is not sufficient to treat most cases of hyperuricemia associated with gout. Within the small number of patients with renal dysfunction, febuxostat was also significantly more effective in achieving the primary efficacy endpoint than allopurinol or placebo. More gout flares were present in the febuxostat $120 \mathrm{mg}$ /day and $240 \mathrm{mg} /$ day arms in the initial 8 weeks of the study, with similar rates among groups in weeks 8-28.

The pattern of adverse events reported in the APEX trial were similar to the ones reported previously in the FACT study, including diarrhea $(6 \%-13 \%$, depending of the febuxostat dose) and liver function tests abnormalities $(4 \%-6 \%)$. In the latter case, the rates were not higher than those found with the fixed dose of allopurinol. Cardiovascular events were registered in similar rates across groups and no deaths during the study period were reported.

Limitations of the FACT study have been pointed out since its publication, and some of these are shared by the APEX trial. An editorial accompanying the FACT trial emphasized that the dose of allopurinol used for comparison (300 mg/day), which could not be modified to preserve the double-blind design, may have been insufficient for a real comparison of the urate-lowering efficacy of these two agents. ${ }^{49}$ Others have commented on the limited generalizability of the trial given the exclusion of patients with kidney dysfunction, a caveat that the APEX trial tried to address with an effort hampered by insufficient recruitment. Some authors pointed towards the need for close monitoring of the safety signals raised by febuxostat in the FACT study, mainly represented by the four deaths in the febuxostat groups. ${ }^{50,51}$ Fortunately, these were not confirmed in the APEX trial. A selection bias towards patients with a previous poor response to allopurinol is a possibility and finally, due to fact that the primary endpoints of this trial were biochemical (reductions in SUA) instead of clinical (ie, disease- rather than patientoriented outcomes), the study is difficult to apply to clinical practice where reductions in gout flares are of paramount interest for patients.

Three ancillary studies published in abstract form only are based on patients studied in these trials. Becker et $\mathrm{al}^{52}$ analyzed patients treated with febuxostat in the 
FACT and APEX studies trying to clarify the relationship between postbaseline SUA, a measure of the amount of SUA reduction after initiation of therapy, and the risk of gout flare. Clear relationships were discerned-early in urate-lowering treatment with the risk of flare being highest in the subjects with the lowest average postbaseline SUA and lowest in those with the highest SUA. This inverse relationship of SUA and flare was demonstrable during the first 3-4 months of treatment but not after 6 months. By 12 months of treatment, the relationship was reversed, so that flare rates were lowest in patients with the lowest SUA. These differences were significant by trend analyses. The same author presented data from 182 African-American patients enrolled in the FACT trial or another 28-week phase III study (APEX) and showed that these patients achieved SUA targets of $6.0 \mathrm{mg} / \mathrm{dL}$ or less in the same proportions as the overall FACT population. Schumacher et al, ${ }^{53}$ using the same base of patients from the FACT and APEX trials, focused on individuals over 65 years old. A total of 295 patients were studied, with reports of good tolerance and efficacy in achieving a target SUA of $6.0 \mathrm{mg} / \mathrm{dL}$ or less in the majority of the population.

The EXCEL study is an open-label phase III extension of the FACT study that, along with additional enrolled patients, is randomizing 735 patients to febuxostat 80 or $120 \mathrm{mg} /$ day or allopurinol $300 \mathrm{mg} /$ day in a $2: 2: 1$ ratio to continue evaluating treatment response. Two interim analyses have been published in abstract form, and report that continuous reduction of SUA to levels of $6.0 \mathrm{mg} / \mathrm{dL}$ or less lead to significant clinical benefits, and that a larger proportion of patients receiving allopurinol treatment compared with febuxostat 80 and $120 \mathrm{mg} /$ day failed to achieve this goal. ${ }^{54,55}$ Finally, abstracts from two Japanese phase III studies reported that febuxostat 20 and $40 \mathrm{mg} /$ day reduced SUA in a dose-dependent manner compared with placebo in 103 patients $^{56}$ and that febuxostat $40 \mathrm{mg} /$ day was more effective in reducing SUA than allopurinol $100 \mathrm{mg}$ twice a day in 256 patients. ${ }^{57}$

In October 2008 the most recent clinical trial of febuxostat was presented at the Annual Meeting of the American College of Rheumatology. This 28-week phase III randomized, controlled, multicenter, double-blind trial comparing efficacy and safety of daily febuxostat and allopurinol in subjects with gout (CONFIRMS) trial randomized 2269 patients to receive febuxostat $40 \mathrm{mg} /$ day, febuxostat $80 \mathrm{mg} / \mathrm{day}$, or allopurinol 200 or $300 \mathrm{mg} /$ day depending on renal function (patients with glomerular filtration rate of $30-59 \mathrm{~mL} / \mathrm{min}$ received $200 \mathrm{mg} /$ day of allopurinol). ${ }^{58}$ The primary endpoint was the proportion of patients achieving an SUA of less than $6 \mathrm{mg} / \mathrm{dL}$ at the end of follow up. A secondary endpoint analyzed this same outcome in those patients with mild or moderate renal dysfunction defined as glomerular filtration rates of 60-89 and 30-59 mL/min, respectively. As in most of the previous febuxostat trials, a majority of the subjects were middle-aged, (mean age 52.8 years) white males. Baseline SUA was $9.6 \mathrm{mg} / \mathrm{dL}$ and gout duration on average was 11.6 years. One thousand four hundred and eighty-three individuals had mild or moderate renal dysfunction. Urate lowering efficacy was similar with allopurinol $300 \mathrm{mg} /$ day (42\% achieved primary endpoint) and febuxostat $40 \mathrm{mg} /$ day (45\% achieved primary endpoint) but febuxostat $80 \mathrm{mg} /$ day was statistically superior to the other two groups (67\% achieved primary endpoint). Among patients with renal dysfunction febuxostat $80 \mathrm{mg} /$ day achieved the primary endpoint in a higher proportion of patients $(72 \%)$ than in those taking febuxostat $40 \mathrm{mg} /$ day $(50 \%)$ or allopurinol (42\%). Rates of adverse events were comparable across groups and at all levels of renal function. Specifically, no difference was reported in the rate of cardiovascular events (six events were reported: three in the allopurinol arm and three in the febuxostat $80 \mathrm{mg}$ /day group). Five deaths occurred during the study, one in each febuxostat group and three in the allopurinol arm. CONFIRMS also provided additional evidence than that provided by the FACT and APEX trials supporting a stronger urate-lowering potency in the tested doses of febuxostat compared with allopurinol for those individuals with SUA greater than $10 \mathrm{mg} / \mathrm{dL}$ or with tophi.

\section{Economic evidence and resource utilization}

No economic studies of febuxostat have been published in peer-reviewed literature. Febuxostat was approved for its marketing in the European Union by the European Medicines Agency in April 2008 under the brand name Adenuric for the indication of "Treatment of chronic hyperuricemia in conditions where urate deposition has already occurred (including a history, or presence of, tophus and/or gouty arthritis). ${ }^{" 59}$ Following this approval the National Institute for Health and Clinical Excellence (NICE) in the United Kingdom released its final appraisal determination for febuxostat in August 2008 ${ }^{60}$ As part of the report, a discussion of an economic analysis performed by the manufacturer of febuxostat was presented, in which febuxostat at doses of 80 and $120 \mathrm{mg}$ /day was compared with allopurinol 
at $300 \mathrm{mg} /$ day over time horizons of 1 and 2 years. The incremental cost-effectiveness ratios were $£ 16574$ (\$US25 920 ) and $£ 15565$ (\$US24 342) per quality-adjusted life-year (QALY), respectively. The ad-hoc committee from NICE questioned this report on several levels based on what they considered to be imprecise estimates and biases. Considering this and the available clinical evidence its approval was finally issued "as an option for the management of chronic hyperuricemia in gout only for people who are intolerant of allopurinol or for whom allopurinol is contraindicated." The manufacturer of febuxostat (Ipsen Pharmaceuticals) filed an appeal to this appraisal that is being processed at time of writing. ${ }^{61}$ Febuxostat was approved for marketing in the US in February 2009 under the brand name, Uloric ${ }^{\circledR}$. Febuxostat's market cost is likely to be substantially higher than its primary competitor, allopurinol, which is available as a generic prescription in the US (\$US8.09 for a 30-day supply of $300 \mathrm{mg} /$ day). Additional costs of febuxostat are still unclear as reports of liver function test abnormalities need further confirmation, but it appears that the usual laboratory monitoring performed with allopurinol to assure that kidney function is stable and that no liver function abnormalities are present will be also necessary with febuxostat. Also, reports about acute gout flares after initiation of febuxostat happening at least as often as with allopurinol makes the expected cost of gout flare prophylaxis the same for both groups, if not somewhat greater with febuxostat.

\section{Patient group/population Allopurinol allergy and intolerance}

In general, allopurinol is usually well tolerated. However, patients with significant toxicities precluding its use have few, if any, alternatives since uricosurics are rarely used in clinical practice and may also be contraindicated in some of these patients. Because febuxostat has a chemical structure unrelated to that of allopurinol, the probability of developing cross-related intolerance to febuxostat is in theory low. Indeed, studies that have reported specifically on allopurinol-intolerant patients have described good results with febuxostat.

\section{Chronic kidney disease}

Uricosurics lose effectiveness in patients with glomerular filtration rates of less than $50 \mathrm{~mL} / \mathrm{min}$, and the frequency of allopurinol adverse effects rises sharply in patients with kidney dysfunction, leading to frequent undertreatment of hyperuricemia in patients with gout and kidney dysfunction. ${ }^{28,62}$ In the few patients with chronic kidney disease studied, febuxostat has had a safe profile with the caveat that, to date, chronic kidney disease patients have been underrepresented in trials. Preliminary results from the CONFIRMS study provided additional information about febuxostat being efficacious and reasonably safe in the short term in patients with moderate kidney dysfunction.

\section{Solid organ transplant}

As described above, patients with solid organ transplants have a higher frequency of hyperuricemia and tophaceous gout. Use of uricosurics and allopurinol may be limited due to comorbidities and multiple drug interactions. To our knowledge, febuxostat has not yet been tested in these patients, but it would represent a welcome addition to the currently limited therapeutic armamentarium in this clinical setting.

\section{Outcomes summary}

Gout continues to significantly affect the quality of life of millions of middle-aged and elderly adults. Its causative metabolic abnormality, hyperuricemia, has been associated with other conditions that are important contributors to the population morbidity and mortality and which prevalence are increasing, like the metabolic syndrome, hypertension and chronic kidney disease. ${ }^{63-65}$ The incidence of both hyperuricemia and gout could also be expected to rise with an aging population with cardiovascular risk factors.

Until February 2009, there were few tenable therapeutic alternatives for gout, and no new drug for its management had been approved in the US since allopurinol in 1964. Uricosuric drugs are of limited application in clinical practice because of inefficacy, adverse effects, and drug interactions. Allopurinol has the main advantage of having been in the market for several decades, having generally good tolerability, low frequency of adverse events, and being rather inexpensive. However, there are patients, including those with chronic kidney disease and solid organ transplantations, in whom allopurinol efficacy is more commonly limited by drug interactions, adverse events, and under dosing. Furthermore, some patients may not tolerate allopurinol and a few may develop hypersensitivity. In these populations and others where hyperuricemia and gout tends to be more prevalent and disabling, a new addition to the treatment armamentarium would be most welcome.

The evidence for the use of febuxostat in gout is summarized in the table at the beginning of this review. Febuxostat, at doses between 80 and $120 \mathrm{mg}$ /day, favorably compares with standard dose allopurinol in reducing SUA. 
Nevertheless, questions still remain over this issue since a double-blind study using as a comparator the commonly used "titration to effect" strategy for allopurinol has not been conducted. There has been a dearth of primary clinical outcomes (gout flares, number or size of tophi) in this first wave of febuxostat studies, as it is expected that the marked SUA-lowering effect translates into clinical improvement in real practice. However, this an important limitation of the phase III studies reported so far for this drug.

Concerns about the safety of febuxostat have been expressed. Initial cardiovascular safety signals raised in the FACT trial have not been replicated in recently released phase III trials. However, postmarketing surveillance will be necessary to monitor for adverse events, given the well-known limitations of clinical trials on this respect.

In conclusion, febuxostat has been approved in the EU and US for the treatment of chronic hyperuricemia in conditions where urate deposition has already occurred, and it will likely find a niche in patients with gout who are unable to use allopurinol because of intolerances, adverse reaction, or drug-drug interactions. In addition, patients with tophaceous gout or with very high SUA levels (more than $10 \mathrm{mg} / \mathrm{dL}$ ) may also benefit from the higher potency of febuxostat versus allopurinol (at the fixed dosages tested). A large proportion of these difficult-to-treat patients have chronic kidney disease and organ transplantations, and they are expected to benefit the most from additional alternatives for the management of chronic gout. However, allopurinol, given its cost and experience, with use will likely remain as the first-line drug for the management of gout in most patients.

\section{Acknowledgements}

Dr Gaffo declares no conflict of interest. Dr Saag has acted as a consultant for, and received research grants from, Takeda Global Research and Development Center, Inc., Savient, Merck.

\section{References}

1. Becker MA, Meenakshi J. Clinical gout and the pathogenesis of hyperuricemia. In: Koopman WJ, Moreland LW, editors. Arthritis and allied conditions: a textbook of rheumatology Philadelphia. Lippincott Williams \& Wilkins; 2005, p. 2 v. (xxiv, 2699, 2108 p).

2. Lawrence RC, Felson DT, Helmick CG, et al. Estimates of the prevalence of arthritis and other rheumatic conditions in the United States: Part II. Arthritis Rheum. 2007;58:26-35.

3. Arromdee E, Michet CJ, Crowson CS, O'Fallon WM, Gabriel SE. Epidemiology of gout: is the incidence rising? J Rheumatol. 2002;29: 2403-2406.
4. Darmawan J, Valkenburg HA, Muirden KD, Wigley RD. The epidemiology of gout and hyperuricemia in a rural population of Java. J Rheumatol. 1992;19:1595-1599.

5. Brauer GW, Prior IA. A prospective study of gout in New Zealand Maoris. Ann Rheum Dis. 1978;37:466-472.

6. Brook RA, Kleinman NL, Patel PA, et al. The economic burden of gout on an employed population. Curr Med Res Opin. 2006;22:1381-1389.

7. Kleinman NL, Brook RA, Patel PA, et al. The impact of gout on work absence and productivity. Value Health. 2007;10:231-237.

8. Singh JA, Strand V. Gout is associated with more comorbidities, poorer health-related quality of life and higher healthcare utilisation in US veterans. Ann Rheum Dis. 2008;67:1310-1316.

9. Stamp L, Searle M, O’Donnell J, Chapman P. Gout in solid organ transplantation: a challenging clinical problem. Drugs. 2005;65: 2593-2611.

10. Campion EW, Glynn RJ, DeLabry LO. Asymptomatic hyperuricemia. Risks and consequences in the normative aging study. Am J Med. 1987;82:421-426.

11. Zhang W, Doherty M, Bardin T, et al. EULAR evidence based recommendations for gout. Part II: Management. Report of a task force of the EULAR Standing Committee for International Clinical Studies Including Therapeutics (ESCISIT). Ann Rheum Dis. 2006a;65:1312-1324.

12. Jordan KM, Cameron JS, Snaith M, et al. British Society for Rheumatology and British Health Professionals in Rheumatology guideline for the management of gout. Rheumatology (Oxford). 2007;46:1372-1374.

13. Emmerson BT. The management of gout. $N$ Engl J Med. 1996;334: 445-451.

14. Mikuls TR, MacLean CH, Olivieri J, et al. Quality of care indicators for gout management. Arthritis Rheum. 2004;50:937-943.

15. Zhang W, Doherty M, Pascual E, et al. EULAR evidence based recommendations for gout. Part I: diagnosis. Report of a task force of the Standing Committee for International Clinical Studies Including Therapeutics (ESCISIT). Ann Rheum Dis. 2006b;65:1301-1311.

16. Borstad GC, Bryant LR, Abel MP, et al. Colchicine for prophylaxis of acute flares when initiating allopurinol for chronic gouty arthritis. J Rheumatol. 2004;31:2429-2432.

17. Pacher P, Nivorozhkin A, Szabo C. Therapeutic effects of xanthine oxidase inhibitors: renaissance half a century after the discovery of allopurinol. Pharmacol Rev. 2006;58:87-114.

18. Day RO, Miners JO, Birkett DJ, et al. Allopurinol dosage selection: relationships between dose and plasma oxipurinol and urate concentrations and urinary urate excretion. Br J Clin Pharmacol. 1988;26:423-428.

19. Dalbeth N, Kumar S, Stamp L, Gow P. Dose adjustment of allopurinol according to creatinine clearance does not provide adequate control of hyperuricemia in patients with gout. $J$ Rheumatol. 2006;33: 1646-1650.

20. Perez-Ruiz F, Alonso-Ruiz A, Calabozo M, et al. Efficacy of allopurinol and benzbromarone for the control of hyperuricaemia. A pathogenic approach to the treatment of primary chronic gout. Ann Rheum Dis. 1998;57:545-549.

21. Riedel AA, Nelson M, Joseph-Ridge N, et al. Compliance with allopurinol therapy among managed care enrollees with gout: a retrospective analysis of administrative claims. J Rheumatol. 2004;31:1575-1581.

22. Owens D, Whelan B, McCarthy G. A survey of the management of gout in primary care. Ir Med J. 2008;101:147-149.

23. Sarawate CA, Patel PA, Schumacher HR, et al. Serum urate levels and gout flares: analysis from managed care data. J Clin Rheumatol. 2006;12:61-65.

24. Halevy S, Ghislain PD, Mockenhaupt M, et al. Allopurinol is the most common cause of Stevens-Johnson syndrome and toxic epidermal necrolysis in Europe and Israel. J Am Acad Dermatol. 2008;58:25-32.

25. Fam AG, Dunne SM, Iazzetta J, Paton TW. Efficacy and safety of desensitization to allopurinol following cutaneous reactions. Arthritis Rheum. 2001;44:231-238. 
26. Young JL Jr, Boswell RB, Nies AS. Severe allopurinol hypersensitivity. Association with thiazides and prior renal compromise. Arch Intern Med. 1974;134:553-558.

27. Lupton GP, Odom RB. The allopurinol hypersensitivity syndrome. J Am Acad Dermatol. 1979;1:365-374.

28. Hande KR, Noone RM, Stone WJ. Severe allopurinol toxicity. Description and guidelines for prevention in patients with renal insufficiency. Am J Med. 1984;76:47-56.

29. Singer JZ, Wallace SL. The allopurinol hypersensitivity syndrome. Unnecessary morbidity and mortality. Arthritis Rheum. 1986;29:82-87.

30. Emmerson BT, Hazelton RA, Frazer IH. Some adverse reactions to allopurinol may be mediated by lymphocyte reactivity to oxypurinol. Arthritis Rheum. 1988;31:436-440.

31. Hung SI, Chung WH, Liou LB, et al. HLA-B*5801 allele as a genetic marker for severe cutaneous adverse reactions caused by allopurinol. Proc Natl Acad Sci U S A. 2005;102:4134-4139.

32. EBPG RT (European Best Practice Guildes Expert Group for Renal Transplantation. European best practice guidelines for renal transplantation. Section IV: long-term management of the transplant recipient. IV.9.2. Haematological complications. Leukopenia. Nephrol Dial Transplant. 2002;17 Suppl 4:49.

33. Fox IH, Kelley WN. Management of gout. JAMA. 1979;242:361-364.

34. Lee MH, Graham GG, Williams KM, Day RO. A benefit-risk assessment of benzbromarone in the treatment of gout. Was its withdrawal from the market in the best interest of patients? Drug Saf. 2008;31:643-665.

35. Terkeltaub R, Bushinsky DA, Becker MA. Recent developments in our understanding of the renal basis of hyperuricemia and the development of novel antihyperuricemic therapeutics. Arthritis Res Ther. 2006; 8(Suppl 1):S4.

36. Hahn P, Edwards N. Management of hyperuricemia. In: Koopman WJ, Moreland LW, editors. Arthritis and allied conditions : a textbook of rheumatology Philadelphia. Lippincott Williams \& Wilkins; 2005, p. 2 v. (xxiv, 2699, 2108 p).

37. Conaghan PG, Day RO. Risks and benefits of drugs used in the management and prevention of gout. Drug Saf. 1994;11:252-258.

38. Izzedine H, Brocheriou I, Becart J, Deray G. Probenecid-induced membranous nephropathy. Nephrol Dial Transplant. 2007;22: 2405-2406.

39. Okamoto K, Eger BT, Nishino T, Kondo S, Pai EF. An extremely potent inhibitor of xanthine oxidoreductase. Crystal structure of the enzyme-inhibitor complex and mechanism of inhibition. $J$ Biol Chem. 2003;278:1848-1855.

40. Becker MA, Schumacher HR, Jr, Wortmann RL, et al. Febuxostat, a novel nonpurine selective inhibitor of xanthine oxidase: a twenty-eightday, multicenter, phase II, randomized, double-blind, placebo-controlled, dose-response clinical trial examining safety and efficacy in patients with gout. Arthritis Rheum. 2005a;52:916-923.

41. Schumacher HR Jr, Becker M, Wortmann RL, et al. Magnetic resonance imaging of gouty tophi during treatment with febuxostat, a non-purine selective inhibitor of xanthine oxidase. Presented at: the Meeting of the American College of Rheumatology; October 16-21, 2004; San Antonio, Texas.

42. Becker M, Schumacher HR Jr, Wortmann RL, et al. Febuxostat, a novel non-purine selective inhibitor of xanthine oxidase, therapy in allopurinol intolerant patients. Presented at: the Meeting of the American College of Rheumatology; October 16-21, 2004; San Antonio, Texas.

43. Wortmann RL, Becker M, Schumacher HR Jr, et al. Gout flare prophylaxis during management of chronic gout with febuxostat, a non-purine selective inhibitor of xanthine oxidase. Poster presented at: 2004; [need full ref].

44. Schumacher HR Jr, Wortmann RL, Becker M, et al. A phase 2, long term open-label safety and efficacy study of febuxostat, a novel non-purine, selective inhibitor of xanthine oxidase. Presented at: the Meeting of the American College of Rheumatology; November 13-17, 2005; San Diego, California.
45. Schumacher HR Jr, Becker M, Wortmann RL, et al. The FOCUS trial 48-month interim analysis: long-term clinical outcomes of treatment with febuxostat in subjects with gout in an ongoing phase 2, open-label extension study. Presented at: the Meeting of the American College of Rheumatology; November 10-15, 2006; Washington, D.C.

46. Kamatani N, Fujimori S, Hada T, et al. Phase II dose-response clinical trial using febuxostat (TMX-67), a novel-type xanthine oxidase/xanthine dehydrogenase inhibitor, for gout and hyperuricemia. Presented at: the Meeting of the American College of Rheumatology; October 23-28, 2003; Orlando, Florida.

47. Becker MA, Schumacher HR Jr, Wortmann RL, et al. Febuxostat compared with allopurinol in patients with hyperuricemia and gout. N Engl J Med. 2005b;353:2450-2461.

48. Schumacher HR Jr, Becker MA, Wortmann RL, et al. Effects of febuxostat versus allopurinol and placebo in reducing serum urate in subjects with hyperuricemia and gout: a 28-week, phase III, randomized, double-blind, parallel-group trial. Arthritis Rheum. 2008;59:1540-1548.

49. Moreland LW. Febuxostat-treatment for hyperuricemia and gout? N Engl J Med. 2005;353:2505-2507.

50. Chatham WW, Saag KG. Is febuxostat a more effective treatment for hyperuricemia and gout than allopurinol? Nat Clin Pract Rheumatol. 2006;2:240-241.

51. Gelber AC. Febuxostat versus allopurinol for gout. $N$ Engl J Med. 2006;354:1532-1533; author reply 1532-1533.

52. Becker M, MacDonald P, Hunt B, Lademacher C, Joseph-Ridge N. Gout flare incidence in relation to average serum urate during the first year of urate-lowering therapy. Presented at: the Meeting of the American College of Rheumatology; November 6-11, 2007a; Boston, Massachusetts.

53. Schumacher HR, Becker M, MacDonald P, et al. Febuxostat versus Allopurinol in the treatment of gout in subjects 65 years of age or older. Presented at: Meeting of The European League Against Rheumatism; June 13-16, 2007; Barcelona, Spain.

54. Wortmann RL, Becker M, Schumacher HR Jr, et al. Effect of febuxostat or allopurinol on the clinical manifestations of gout: reduction in gout flares and tophus size over time in the EXCEL trial. Presented at: the Meeting of the American College of Rheumatology; November 10-15, 2006; Washington, D.C.

55. Becker M, Schumacher HR Jr, MacDonald P, et al. Urate-lowering therapy (febuxostat [FEB] or allopurinol [ALLO]) in subjects with gout: interim results from the febuxostat comparative extension long-term study (EXCEL). Presented at: the Meeting of the American College of Reumatology; Novemeber 6-11, 2007b; Boston, Massachusetts.

56. Kamatani N, Fujimori S, Hada T, et al. Febuxostat, a novel non-purine selective inhibitor of xanthine oxidase, in a phase III placebo-controlled double-blind clinical trial in Japanese subjects with gout or hyperuricemia. Presented at: the Meeting of the American College of Rheumatology; October 16-21, 2004a; San Antonio, Texas.

57. Kamatani N, Fujimori S, Hada T, et al. Febuxostat, a novel non-purine selective inhibitor of xanthine oxidase, in an allopurinol-controlled phase III clinical trial in Japanese subjects with gout or hyperuricemia. Presented at: the Meeting of the American College of Rheumatology; October 16-21, 2004b; San Antonio, Texas.

58. Becker M, Schumacher HR Jr, Espinoza L, et al. A phase 3 randomized, controlled, multicenter, double-blind trial comparing efficacy and safety of daily febuxostat and allopurinol in subjects with gout. Presented at: the Meeting of the American College of Rheumatology; October 24-29, 2008; San Francisco, California.

59. EMA (European Medicines Agency). Available at: http://www.emea. europa.eu/humandocs/Humans/EPAR/adenuric/adenuric.htm (accessed November 10, 2008).

60. NICE (National Institute of Health and Clinical Excellence) 2008a. Available at: http://www.nice.org.uk/nicemedia/pdf/ STAFebuxostatGoutFAD.pdf (accessed November 10, 2008).

61. NICE (National Institute of Health and Clinical Excellence) 2008b. Available at: http://www.nice.org.uk/guidance/index.jsp?action=fold er\&o=42356 (accessed November 10, 2008). 
62. Dalbeth N, Stamp L. Allopurinol dosing in renal impairment: walking the tightrope between adequate urate lowering and adverse events. Semin Dial. 2007;20:391-395.

63. Ford ES, Giles WH, Dietz WH. Prevalence of the metabolic syndrome among US adults: findings from the third National Health and Nutrition Examination Survey. JAMA. 2002;287:356-359.
64. Kang DH, Nakagawa T, Feng L, et al. A role for uric acid in the progression of renal disease. J Am Soc Nephrol. 2002;13:2888-2897.

65. Masuo K, Kawaguchi H, Mikami H, Ogihara T, Tuck ML. Serum uric acid and plasma norepinephrine concentrations predict subsequent weight gain and blood pressure elevation. Hypertension. 2003;42:474-480.

\section{Publish your work in this journal}

Core Evidence is an international, peer-reviewed open-access journal evaluating the evidence underlying the potential place in therapy of drugs throughout their development lifecycle from preclinical to postlaunch. The focus of each review is to evaluate the case for a new drug or class in outcome terms in specific indications and patient

\section{Dovepress}

groups. The manuscript management system is completely online and includes a very quick and fair peer-review system, which is all easy to use. Visit http://www.dovepress.com/testimonials.php to read real quotes from published authors. 